\title{
Biventricular apical ballooning in patient with COVID-19
}

\author{
Azin Alizadehas ${ }^{1} \cdot$ Azam Soleimani $^{2}{ }^{\circledR} \cdot$ Mohammad Mehdi Peighambari $^{3} \cdot$ Atousa Mostafavi $^{4}$
}

Received: 7 January 2021 / Revised: 20 March 2021 / Accepted: 30 April 2021 / Published online: 12 May 2021

(c) Japanese Society of Echocardiography 2021

COVID-19 disease due to severe acute respiratory syndrome Coronavirus 2 (SARS- $\mathrm{CoV}_{2}$ ) has different types of cardiovascular manifestations. Detectable high sensitivity cardiac troponin I and abnormal CMR has been reported in $71 \%$ and $78 \%$ of recently recovered COVID-19 patients, respectively [1]. Cardiac injury associated with COVID-19 illness has been attributed to cytokine storm, viral myocarditis as well as ischemia due to microvascular and macrovascular dysfunction [2].

Herein, we present a 57-year-old male, known case of hypertension and dyslipidemia, under treatment with losartan and atorvastatin, presented with dyspnea and dry cough for 5 days. Low-grade fever (oral temperature of $37.9^{\circ} \mathrm{C}$ ), stable vital signs without hypoxemia or respiratory distress, was observed, so he was discharged after describing alarm signs. After 10 days, he presented with complaint of retrosternal chest pain and dyspnea for 2 days. He was afebrile, ill and agitated with hypoxemia $\left(\mathrm{SPaO}_{2}\right.$ of $\left.77 \%\right)$, tachypnea (respiratory rate: 28/min), tachycardia (HR:115 bpm), hypotension (blood pressure: 90/65), cold sweat and rales in half of the lung fields, representing acute pulmonary edema. Electrocardiogram revealed sinus tachycardia with mild dynamic ST segment depression in precordial leads. Oxygen therapy via nasal mask, intravenous furosemide, inotrope, anticoagulant, and oral antiplatelets was administered while chest CT scan demonstrated ground glass opacities

Azam Soleimani

asoleimanii@gmail.com

1 Cardio-Oncology Department and Research Center, Rajaie Cardiovascular Medical and Research Center, Iran University of Medical Sciences, Tehran, Iran

2 Cardiac Rehabilitation Research Center, Isfahan Cardiovascular Research Institute, Isfahan University of Medical Sciences, Isfahan, Iran

3 Interventional Cardiology Research Center, Rajaie Cardiovascular Medical and Research Center, Iran University of Medical Sciences, Tehran, Iran

4 Tehran University of Medical Sciences, Shariati Hospital, Tehran, Iran compatible with COVID-19 and congestion (Fig. 1a). No significant coronary artery stenosis was observed during catheterization (Fig. 1b), but transient bradycardia led to temporary pace maker insertion. Transthoracic echocardiography showed akinesia in mid-to-apical segments of both ventricles compatible with biventricular apical ballooning syndrome (Takotsubo cardiomyopathy) (Fig. 1c,d, Supplementary Video 1). Laboratory data showed positive oropharyngeal swab for CoV-2, lymphopenia (952 per $\mu \mathrm{L})$ without leukocytosis $(6800$ per $\mu \mathrm{L})$, elevated cardiac troponin I $(183 \mathrm{ng} / \mathrm{L}$, normal < 50) and C-reactive protein $(18.9 \mathrm{mg} / \mathrm{L}$, normal $<5)$. His clinical course was unremarkable with mild biventricular residual systolic dysfunction on 6 week follow-up.

Stress cardiomyopathy also known as Takotsubo cardiomyopathy or apical ballooning syndrome is known as acute coronary syndrome presentation accompanied by dynamic ST-segment changes and myocardial dysfunction in a noncoronary distribution (3). Takotsubo cardiomyopathy has been reported during SARS- $\mathrm{CoV}_{2}$ infection in the setting of preexisting coronary artery disease along with normal coronary angiogram [3, 4]. Male predominance was proposed in Takotsubo cardiomyopathy associated with COVID-19, despite usual forms of stress cardiomyopathy [5].

This biventricular variant of Takotsubo cardiomyopathy in a male patient is a unique form of cardiac injury during COVID-19 disease with favorable outcome.

Supplementary Information The online version contains supplementary material available at https://doi.org/10.1007/s12574-021-00530-z.

\section{Declarations}

Conflict of interest Azin Alizadehasl, Azam Soleimani, Mohammad Mehdi Peighambari, Atousa Mostafavi declare that they have no conflict of interest.

Human rights statements All procedures followed were in accordance with the ethical standards of the responsible committee on human experimentation (institutional and national) and with the Helsinki Declaration of 1964 and later versions. 


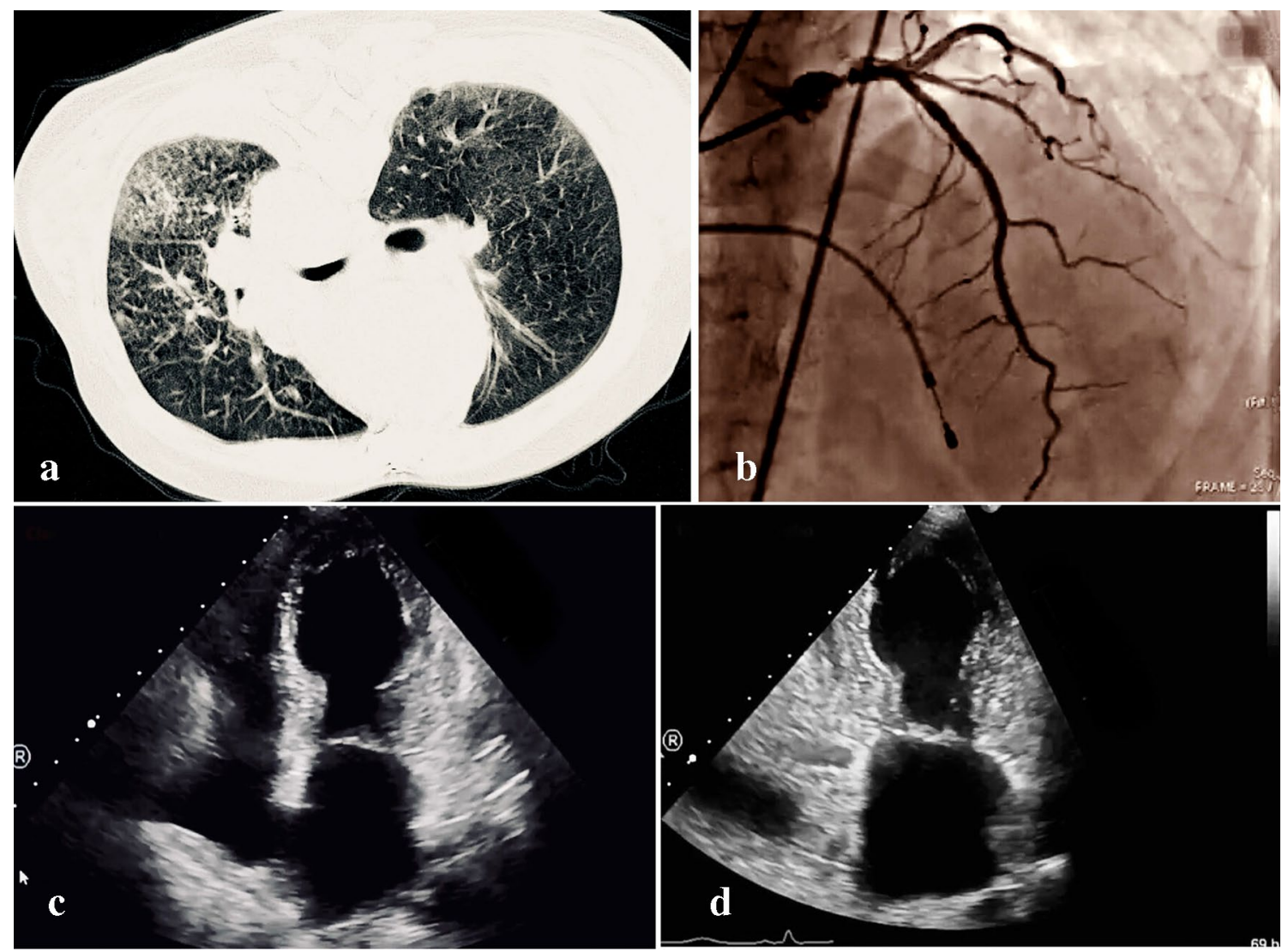

Fig. 1 a Mid thoracic slice of chest CT scan showed ground glass peripheral opacities accompanied by congested lung tissue. b Left coronary angiography revealed normal epicardial coronary arteries,

Informed consent Informed consent was obtained from the patient for being included in the study.

\section{References}

1. Puntmann VO, Carerj ML, Wieters I, et al. Outcomes of cardiovascular magnetic resonance imaging in patients recently recovered from Coronavirus disease, COVID-19. JAMA Cardiol. 2019;2020:3557.

2. Hendren NS, Drazner MH, Bozkurt B, et al. Description and proposed management of the acute COVID-19 cardiovascular syndrome. Circulation. 2020;141(23):1903-14. pace maker lead is seen in RV apex too. $\mathbf{c}, \mathbf{d}$ Transthoracic apical four and two chamber views show akinesia in mid to apex of both ventricles associated with ballooning appearance

3. Minhas AS, Scheel P, Garibaldi B, et al. Takotsubo syndrome in the setting of COVID-19. J Am Coll Cardiol Case Rep. 2020;2(9):1321-5.

4. Tsao CW, Strom JB, Chang JD, et al. COVID-19 associated stress (Takotsubo) cardiomyopathy. Circulation. 2020;13(7):e011222.

5. Giustino G, Croft LB, Oates CP, et al. Takotsubo cardiomyopathy in COVID-19. JACC. 2020;76(5):628-9.

Publisher's Note Springer Nature remains neutral with regard to jurisdictional claims in published maps and institutional affiliations. 